\title{
Pentoxifylline inhibits TLR- and inflammasome-mediated in vitro inflammatory cytokine production in human blood with greater efficacy and potency in newborns
}

\author{
Esther M. Speer ${ }^{1}$, David J. Dowling ${ }^{2,3}$, Lukasz S. Ozog ${ }^{1}$, Jianjin Xu' ${ }^{4}$, Jie Yang ${ }^{5}$, Geetika Kennady ${ }^{1}$ and Ofer Levy ${ }^{2,3,6}$
}

BACKGROUND: Toll-like receptor (TLR)-mediated inflammation may contribute to neonatal sepsis, for which pentoxifylline (PTX), a phosphodiesterase inhibitor that raises intracellular CAMP, is a candidate adjunctive therapy. We characterized the anti-inflammatory effects of PTX toward TLR-mediated production of inflammatory (tumor necrosis factor (TNF) and interleukin (IL)-1 $\beta$ ) and proresolution (IL-6 and IL-10) cytokines in human newborn and adult blood.

METHODS: Newborn cord and adult blood were treated with PTX (50-400 $\mu \mathrm{mol} / \mathrm{l})$ before, during or after stimulation with LPS (TLR4 agonist), R848 (TLR7/8 agonist) or LPS/ATP (inflammasome activation). Cytokines were measured by multiplex assay (supernatants), intracellular cytokines and signaling molecules by flow cytometry, and mRNA by quantitative real-time PCR.

RESULTS: Whether added $2 \mathrm{~h}$ pre-, simultaneously to, or $2 \mathrm{~h}$ post-TLR stimulation, PTX inhibited TLR-mediated cytokine production in a concentration-dependent manner, with greater efficacy and potency in newborn blood, decreasing intracellular TNF and IL-1 $\beta$ with relative preservation of IL-10 and IL-6. PTX decreased TLR-mediated TNF mRNA while increasing IL-10 mRNA. Neonatal plasma factors contributed to the anti-inflammatory effects of PTX in newborn blood that were independent of soluble TNF receptor concentrations, p38 MAPK phosphorylation and IKB degradation.

CONCLUSION: PTX is a potent and efficacious inhibitor of TLR-mediated inflammatory cytokines in newborn cord blood and a promising neonatal anti-inflammatory agent.

$\mathbf{T}$ he neonatal inflammatory response to perinatal infections is associated with increased mortality and major morbidity such as sepsis, bronchopulmonary dysplasia (BPD), and cerebral palsy $(1,2)$. Corticosteroids, the current anti-inflammatory treatment of choice for neonates, have significant short-term (e.g., insulin resistance) and long-term (e.g., growth restriction and neurodevelopmental disability) adverse effects $(3,4)$. There is thus an unmet clinical need for effective anti-inflammatory alternatives to corticosteroids with less adverse effects to treat neonatal inflammation.

The xanthine derivative pentoxifylline (PTX) is a candidate anti-inflammatory agent. PTX competitively inhibits phosphodiesterases in a range of cells and tissues, thereby increasing intracellular CAMP, a second messenger which acts via protein kinase A to suppress gene transcription of proinflammatory mediators including tumor necrosis factor (TNF) $(5,6)$. Due to its anti-TNF activity, PTX has been studied in animals and human adults and newborns to ameliorate inflammatory conditions such as sepsis, BPD, meconium aspiration, and hypoxic ischemic encephalopathy (7-11). Adverse reactions of PTX in adults include gastrointestinal and CNS symptoms, whereas significant adverse reactions at therapeutic doses are rare in adults (www.pdr.net/drug-summary/Pentoxifylline, www.fda. gov). Although recent cerebral and/or retinal hemorrhage are contraindications in adults (www.pdr.net/drug-summary/ Pentoxifylline), intraventricular hemorrhage and bleeding diathesis have not been reported in septic preterm neonates treated with PTX $(7,12)$. A recent Cochrane Review concluded that the use of PTX as an adjunct to antibiotics in neonatal sepsis may decrease all-cause mortality without any adverse effects (12).

Although preliminary studies suggest that PTX may have utility as an anti-inflammatory agent in vivo, much remains to be learned about its action and the range of pattern recognition receptor pathways it may inhibit. A more comprehensive characterization of the action of PTX with respect to (i) Tolllike receptor (TLR)- and/or inflammasome-mediated cytokine induction and (ii) its relative potency and efficacy with age may help inform further development of this promising agent, especially as clinical characteristics of sepsis are very distinct with age (13). Consistent with the sensitivity of newborn leukocytes to cAMP axis inhibition (14) and to the known signaling of endotoxin via TLR4, we hypothesized that PTX may decrease TLR-mediated cytokine production with higher efficacy in newborns as compared with adults. As a model for infection-induced neonatal inflammation, we stimulated newborn cord and adult

\footnotetext{
'Department of Pediatrics, Stony Brook University School of Medicine, Stony Brook, New York; ${ }^{2}$ Department of Medicine, Division of Infectious Diseases, Boston Children's Hospital, Boston, Massachusetts; ${ }^{3}$ Harvard Medical School, Boston, Massachusetts; ${ }^{4}$ Department of Applied Mathematics and Statistics, Stony Brook University School of Medicine, Stony Brook, New York; ${ }^{5}$ Family, Population, and Preventive Medicine Department, Stony Brook University School of Medicine, Stony Brook, New York; ${ }^{6}$ Precision Vaccines Program, Boston Children's Hospital, Boston, Massachusetts. Correspondence: Esther M. Speer (esther.speer@stonybrookmedicine.edu)

Received 14 July 2016; accepted 19 November 2016; advance online publication 22 March 2017. doi:10.1038/pr.2017.6
} 
peripheral whole blood with TLR4 (LPS or endotoxin), TLR7/8 (R848, an imidazoquinoline that induces adult-level inflammatory cytokines in newborn monocytes) (15), or combined TLR and inflammasome activators including R848 (15) and (LPS and ATP). This approach has the advantage of preserving the antiinflammatory factors naturally present in newborn plasma and erythrocytes $(14,16,17)$, thus more accurately modeling pathophysiologically relevant conditions (18). Furthermore, our study also broadened the range of innate signaling pathways studied with respect to PTX sensitivity, including the TLR7/8 pathway important to recognition of bacterial and viral single stranded RNAs $(19,20)$. Using these whole blood assays, we compared the effects of PTX on TLR-mediated cytokine mRNA expression, intracellular cytokines, and supernatant cytokine concentrations in term cord blood and adult peripheral blood. In order to investigate the underlying mechanisms involved, we examined the modulatory effects of neonatal plasma on the anti-inflammatory efficacy of PTX and dibutyryl (db)-cAMP. Our findings demonstrated that PTX, enhanced by neonatal plasma factors and independently of p38 mitogen activated protein kinase (MAPK) phosphorylation or inhibitory kappa B (IkB) degradation, inhibited inflammatory cytokine release with higher efficacy and potency in the newborn while preserving endogenous interleukin (IL)-6 and IL-10 production. PTX might thus provide a promising alternative anti-inflammatory therapy for prevention and/or treatment of inflammatory neonatal conditions.

\section{METHODS}

\section{Subjects and Human Blood Collection}

Collection of placental cord blood and adult peripheral venous blood was approved by the Institutional Review Board of Stony Brook University, Stony Brook, NY, and written informed consent was obtained from participating subjects. Healthy term newborns between 38 and $41 \mathrm{wk}$ gestation delivered at Stony Brook University Medical Center by Cesarean section without labor and without documented intrauterine infection were eligible. Nonexposure to intrauterine infection was defined as absence of clinical chorioamnionitis, fetal membrane rupture over $12 \mathrm{~h}$, meconium-stained amniotic fluid, clinical or laboratory signs of early-onset sepsis, or culture-proven sepsis of the mother or the newborn. Clinical chorioamnionitis was defined as maternal temperature $\geq 37.8^{\circ} \mathrm{C}$ intrapartum or up to $2 \mathrm{~h}$ after delivery, and two or more of the following signs: uterine tenderness, purulent or foul-smelling vaginal discharge, maternal or fetal tachycardia, or maternal leukocytosis (21). Cord blood was collected immediately after delivery of the placenta into sodium heparin tubes containing 15 units/ml heparin (Becton Dickinson, Franklin Lakes, NJ) through puncture of the veins on the fetal side of the placenta using sterile techniques, as previously described (22).

Exclusion criteria comprised pre-existing conditions such as diabetes and autoimmune diseases, and pregnancy complications including pre-eclampsia, fetal chromosomal or major congenital anomalies, intrauterine growth restriction, asphyxia, and life-threatening maternal or fetal conditions potentially impacting the immune response. Healthy adult blood donors between 18 and $55 \mathrm{y}$ of age were recruited at Stony Brook University. Upon subject enrollment, basic demographic, medical, and obstetric data was collected through an interview with the participant to verify eligibility. Exclusion criteria consisted of any medical condition that may affect the subjects' immune response such as pregnancy, diabetes, cancer, or autoimmune disease, and any infection within the past month.

\section{In Vitro Stimulation and Treatment of Blood Samples}

Samples were kept at room temperature and processed within $2 \mathrm{~h}$. Blood was diluted $1: 1$ with sterile prewarmed $\left(37^{\circ} \mathrm{C}\right)$ Roswell Park
Memorial Institute (RPMI) 1640 (Life Technologies, Grand Island, NY) and a final volume of $200 \mu \mathrm{l}$ was added to each well of 96-well tissue culture-treated round-bottom polystyrene plates (Becton Dickinson). Samples were treated with PTX (Tocris, Minneapolis, $\mathrm{MN}$ ) or vehicle control, stimulated with TLR agonists, and cultured at $37^{\circ} \mathrm{C}$ in a humidified incubator at $5 \% \mathrm{CO}_{2}$. Ultrapure preparation of LPS (LPS from E. coli O111:B4 ultrapure, InvivoGen, San Diego, CA) was used for TLR4 stimulation, R848 (InvivoGen) for TLR7/8 stimulation, or LPS followed by $5 \mathrm{mmol} / \mathrm{l}$ ATP (Sigma-Aldrich, St. Louis, MO) for $20 \mathrm{~min}$ for inflammasome induction. Upon completion, samples were centrifuged in situ at $500 \mathrm{~g}$ for $10 \mathrm{~min}$ at room temperature. Approximately $120 \mu \mathrm{l}$ supernatants per well were carefully collected without disturbing the cell pellets and stored at $-80^{\circ} \mathrm{C}$ until analyzed. Duplicate technical replicates were used for all culture experiments and immunoassays, whereas real time PCR experiments were performed in triplicates. The number of independently conducted experiments utilizing blood samples from different donors was as indicated for each experiment. The optimal duration of TLRagonist stimulation for the different experimental procedures was determined through prior kinetic studies.

\section{Measurement of Cytokine Concentrations in Culture Supernatants}

Supernatant cytokine concentrations were determined with Bio-Plex Pro magnetic multiplex assays (Bio-Rad, Hercules, CA) and analyzed on the Bio-Plex 200 system with Bio-Plex Manager 5.0 software (Bio$\mathrm{Rad})$. Cytokine concentrations were expressed in percent compared to TLR agonists alone, defined as $100 \%$.

\section{Real Time PCR}

Total RNA was isolated from cultured whole blood after erythrocyte lysis using QIAamp RNA Blood Mini kits (Qiagen, Valencia, CA). Genomic DNA was removed with RNase-free DNase (Qiagen). Reverse transcription employed High Capacity cDNA Reverse Transcription kits (Life Technologies, Foster City, CA). Real-time PCR was performed using TaqMan gene expression assays (Life Technologies). In order to minimize the possibility of genomic DNA expression, probes that span exon junctions were chosen whenever available from the manufacturer. No reverse transcription controls were used to confirm the absence of genomic DNA amplification for all gene expression assays. Beta actin served as normalization control and was multiplexed into all reaction wells. In order to assure noninterference of the housekeeping gene with the target genes, all gene expression assays were first pretested by comparing their amplification efficiencies as single-plex assay with their amplification efficiencies when multiplexed with the housekeeping gene. Reactions were run on a StepOne Plus Real-time PCR system (Life Technologies) using TaqMan Fast Advanced Master Mix (Life Technologies). Data were analyzed using the delta-delta threshold cycle $\left(\Delta \Delta \mathrm{C}_{\mathrm{T}}\right)$ method (23).

\section{Flow Cytometry}

Samples were prepared with the Cytofix/Cytoperm Fixation/ Permeabilization kit (BD Biosciences, San Jose, CA) with (TNF, IL-6, and IL-10) or without (IL-1 $\beta$ ) addition of Brefeldin A. After pretreatment with PTX or vehicle control for $2 \mathrm{~h}$, samples were stimulated with TLR agonists and cultured at $37^{\circ} \mathrm{C}$ in $5 \% \mathrm{CO}_{2}$ for $6 \mathrm{~h}$. Following surface staining, red blood cell lysis employed FACS Lysing Solution (BD). After fixation and permeabilization, samples were stained with monoclonal antibodies (PE-Cy7-conjugated mouse anti-TNF/ clone MAb11, PE-conjugated mouse anti-IL-6/clone AS12, APCconjugated rat anti-IL-10/clone JES3-19F1, PE-conjugated mouse anti-IL-1 $\beta /$ clone AS10) or their corresponding isotype controls. Samples were analyzed on an LSR Fortessa flow cytometer (BD). Compensation beads (BD Comp Beads, BD) were used as single-stain positive and negative controls. Monocytes were gated with forward and side scatter as $\mathrm{CD} 45^{+} / \mathrm{CD} 14^{+}$cells (PerCP-Cy5.5-conjugated mouse anti-CD45/clone 2D1, FITC-conjugated mouse anti-CD14/ clone M5E2). Data from 10,000 monocytes were acquired for each condition and analyzed with Kaluza version 1.3 software (Beckman Coulter, Jersey City, NJ). The geometric mean fluorescence intensity of all monocytes was determined after subtraction of isotype controls. 


\section{Articles | speretal.}

Samples for measurements of p38 MAPK phosphorylation and total IKB were pretreated with PTX or vehicle control for $2 \mathrm{~h}$ and stimulated with TLR agonists for $15 \mathrm{~min}$, followed by immediate red blood cell lysis and fixation using Lyse/Fix Buffer (BD). After permeabilization with Perm Buffer II (BD), samples were simultaneously stained with the appropriate surface and intracellular antibodies (AF647-conjugated mouse anti-p38(pT180/pY182)/clone 36 or AF647-conjugated mouse anti-IkB $\alpha /$ clone 25/IkB $\alpha / \mathrm{MAD}-3$ ).

\section{Isolation of Hemocytes and Resuspension in Homologous and Heterologous Plasma}

As previously described (14), neonatal and adult hemocytes were separated from plasma after centrifugation at $1,000 \mathrm{~g}$ in room temperature for $10 \mathrm{~min}$. Plasma samples were further purified by centrifugation at $3,000 \mathrm{~g}$ for $10 \mathrm{~min}$. Hemocytes were washed three times in Hank's Buffered Salt solution, and resuspended in autologous or heterologous plasma.

\section{Endotoxin Testing}

All immune-modulators and TLR agonists except LPS were verified endotoxin-free $(<0.1 \mathrm{EU} / \mathrm{ml})$ by the Limulus amebocyte lysate assay (Charles River, Wilmington, MA).

\section{Statistical Analysis}

Linear mixed models for dose response data were employed to compare supernatant and intracellular concentrations for each cytokine between subject cohorts. The covariance structure among drug concentrations within a subject was modeled as compound symmetry. To ensure residual normality, cytokine values and drug concentrations may be log transformed as indicated. For gene expression data, a linear mixed model was employed to analyze the $\Delta \Delta C_{\mathrm{T}}$ values for each gene independently. The covariance between two time points within a subject was modeled as unstructured variance, and the covariance among different stimulations and treatments within a time point was modeled as compound symmetry. SAS 9.3 (SAS Institute, Cary, NC) statistical software was used for analyses and GraphPad Prism Version 6.01 (GraphPad Software, San Diego, CA) for graphing of results. Due to the exploratory nature of this study, no multiple testing adjustments were implemented (24). All statistical tests were twosided and findings considered significant at $P<0.05$.

\section{RESULTS}

\section{Compared With Adult Blood, Newborn Cord Blood}

Demonstrated Diminished LPS-induced TNF but Elevated IL-10

Production

In line with previous published reports $(25,26)$, newborn blood demonstrated diminished LPS-induced TNF production but comparable IL-6, IL-1 $\beta$, and R848-induced TNF production to adult blood. By contrast, LPS-, R848-, and LPS/ ATP-induced IL-10 concentrations were elevated in newborn blood (Figure 1). These results indicate that our in vitro whole blood assay system modeled the typical age and TLR-pathway pattern described for newborns (27).

\section{PTX Inhibited TLR-mediated Inflammatory Cytokine Production} with Higher Efficacy and Potency in Newborn Blood

Except for a modest inhibition of basal IL-1 $\beta$ at high concentration $(400 \mu \mathrm{mol} / \mathrm{l})$, PTX added alone did not affect cytokine production in unstimulated human blood (see Supplementary Figure $\mathbf{S 1}$ online).When added $2 \mathrm{~h}$ prior to TLR stimulation,

b
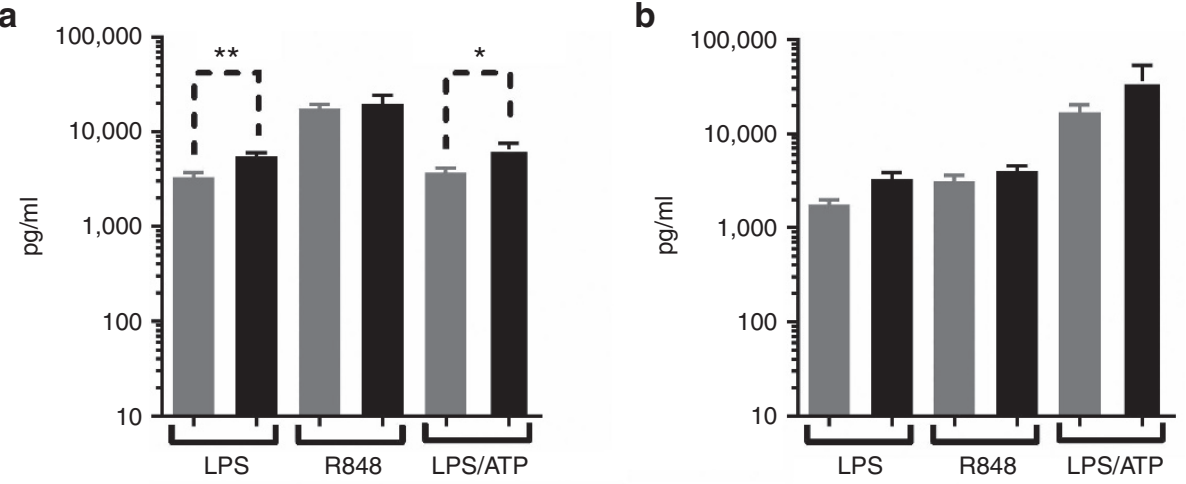

C

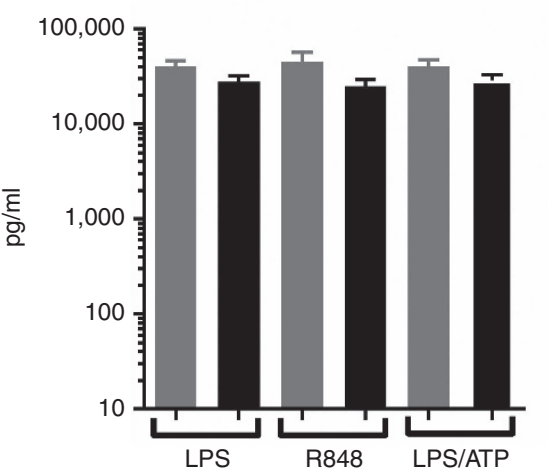

d

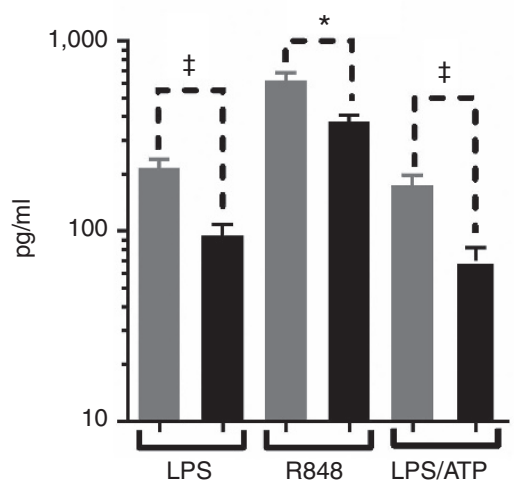

Figure 1. Distinct TLR- and/or inflammasome-mediated cytokine production in human cord and adult blood. Cord blood from healthy term newborns ( $n$ $=10$ ) and peripheral blood from healthy adults $(n=10)$, diluted $1: 1$ with RPMI 1640 , was stimulated with $10 \mathrm{ng} / \mathrm{ml} \mathrm{LPS,} 1 \mu \mathrm{g} / \mathrm{ml} \mathrm{R848}$ or LPS followed by $5 \mathrm{mmol} / \mathrm{I}$ ATP for $20 \mathrm{~min}$ for inflammasome induction. Samples were cultured for $6 \mathrm{~h}$ at $37^{\circ} \mathrm{C}$ in $5 \% \mathrm{CO}_{2}$. Mean ( \pm SEM) supernatant (a) TNF, (b) IL- $1 \beta$, (c) IL- 6 , and (d) IL-10 concentrations for newborns $(\square)$ and adults $(\square)$ are shown. Group comparisons employed t-tests, and significant differences were indicated: ${ }^{*} P<0.05,{ }^{* *} P<0.01$, and ${ }^{\ddagger} P<0.001$. ATP, adenosine triphosphate; IL, interleukin; RPMI, Roswell Park Memorial Institute; TNF, tumor necrosis factor; TLR, Toll-like receptor. 
a

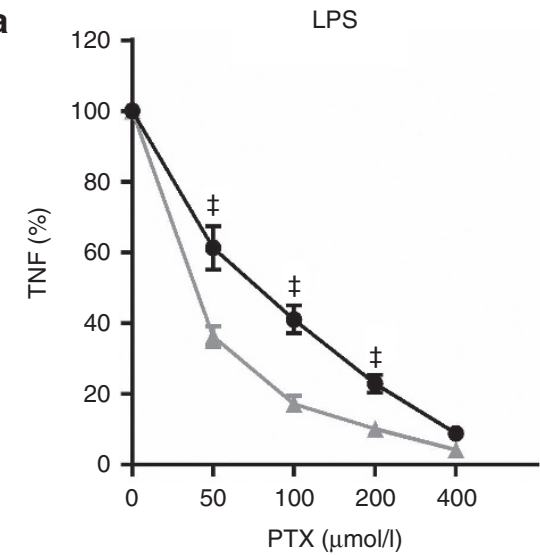

b

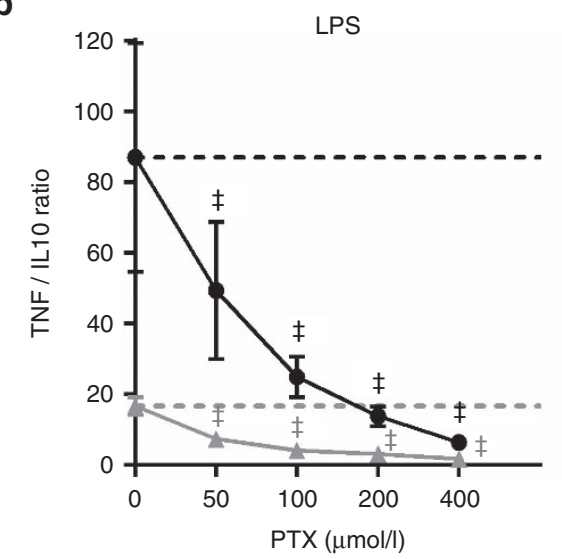

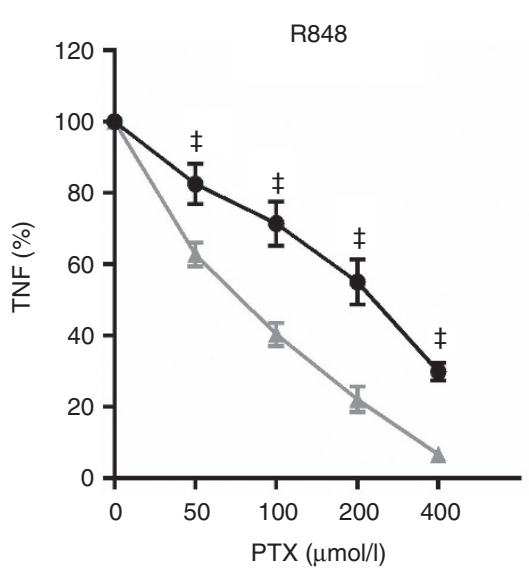

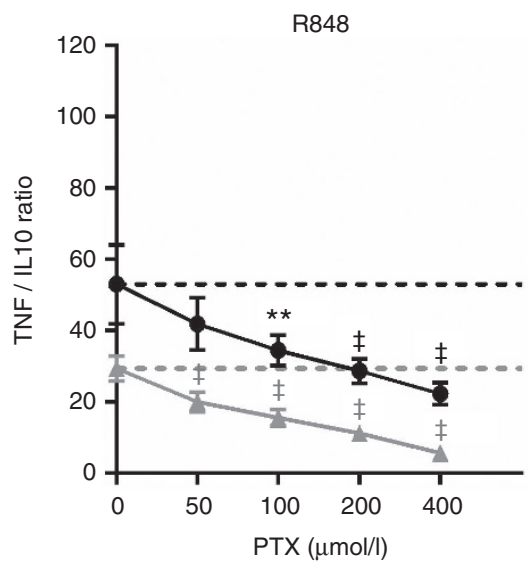

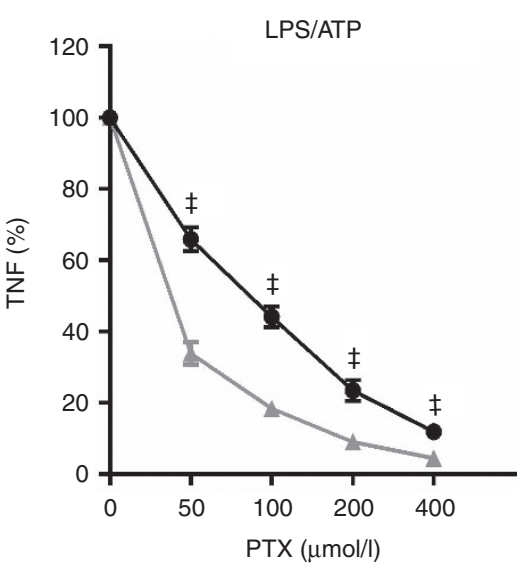

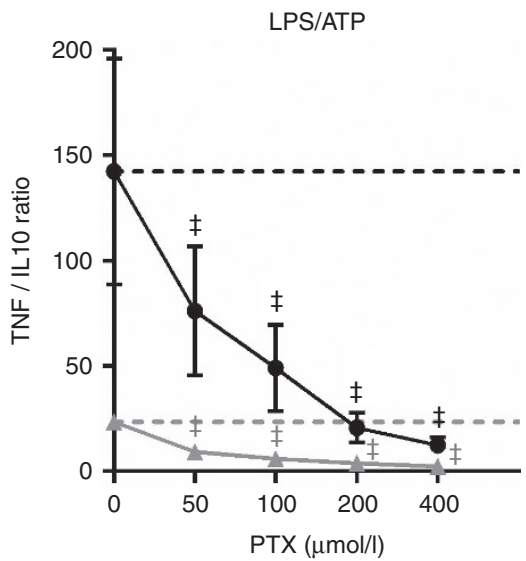

Figure 2. PTX inhibited TLR- and/or inflammasome-mediated TNF production with higher efficacy in newborn blood. Cord ( $n=10)$ and adult blood ( $n$ $=10$ ), diluted $1: 1$ with RPMI 1640, was pretreated with PTX or vehicle control for $2 \mathrm{~h}$. Samples were stimulated with $10 \mathrm{ng} / \mathrm{ml} \mathrm{LPS,} 1 \mu \mathrm{g} / \mathrm{ml} \mathrm{R848}$ or LPS followed by $5 \mathrm{mmol} / \mathrm{I}$ ATP for $20 \mathrm{~min}$ for inflammasome activation, and cultured for $6 \mathrm{~h}$ at $37^{\circ} \mathrm{C}$ in $5 \% \mathrm{CO}_{2}$. Supernatant cytokine concentrations were measured, and results expressed in percent compared to TLR agonists alone, defined as $100 \%$. (a) Mean ( \pm SEM) TLR-mediated TNF concentrations in newborn $(\boldsymbol{\Lambda})$ vs. adult blood $(\bullet)$ in response to equimolar PTX. Data were analyzed with linear mixed models, and significant differences between newborn and adult samples at equimolar PTX concentrations were indicated: ${ }^{*} P<0.05,{ }^{* *} P<0.01$, and ${ }^{\ddagger} P<0.001$. (b) Mean ( \pm SEM) TNF-to-IL-10 concentration ratios for newborn ( $\mathbf{\Delta}$ ) and adult samples $(\bullet)$ plotted against PTX concentrations. Significant decreases in TNF-to-IL-10 ratios of PTX-treated newborn (gray signs) and adult (black signs) blood compared with the corresponding untreated newborn and adult control samples (gray and black dotted lines, respectively) were indicated. ATP, adenosine triphosphate; IL, interleukin; RPMI, Roswell Park Memorial Institute; PTX, pentoxifylline; TNF, tumor necrosis factor; TLR, Toll-like receptor.

PTX inhibited TLR agonist-mediated inflammatory cytokines in a concentration-dependent manner. Employing linear mixed models, PTX exerted significantly greater inhibition of LPSinduced TNF compared with IL-1 $\beta$ production, and significantly greater inhibition of TLR4 (LPS)- than TLR7/8 (R848)-induced TNF in newborn or adult blood (Figure 2a, Supplementary Figure S2 online). Consistent with our hypothesis, PTX demonstrated significantly greater inhibition of LPS-, R848-, and inflammasome-induced TNF production in newborn compared with adult blood (Figure 2a, Table 1) and greater inhibition of R848-induced IL-1 $\beta$ and IL-6 production in newborn blood (see Supplementary Figure S2 online). Most newborn samples demonstrated greater inhibition of TLR-mediated TNF across all drug concentrations (see Supplementary Figure S3 online). Nonlinear regression with robust fit (GraphPad Prism) was used to estimate the inhibitory concentration $50\left(\mathrm{IC}_{50}\right)$ of PTX for LPS-induced TNF production, which was $49.8 \mu \mathrm{mol} / \mathrm{l}$ in newborn vs. $93.5 \mu \mathrm{mol} / \mathrm{l}$ in adult blood.
PTX decreased TLR-mediated TNF at all tested concentrations in newborn and adult blood (Table 1), albeit with higher efficacy in newborns (Figure 2a). Whereas inhibition of LPS/ ATP- and R848-induced IL-1 $\beta$ release was only achieved with concentrations $\geq 100 \mu \mathrm{mol} / \mathrm{l}$ in adult blood, PTX decreased LPS-, R848-, and LPS/ATP-induced IL-1 $\beta$ at all tested concentrations including $50 \mu \mathrm{mol} / \mathrm{l}$ in newborn blood (Table 1). This indicates that PTX inhibits TLR agonist-mediated IL- $1 \beta$ with higher potency in the newborn. While PTX decreased LPS-, R848-, and LPS/ATP-induced IL-6 at all tested concentrations in newborn samples, it did not affect R848-induced IL-6 in adult blood (Table 1).

\section{PTX Decreased the TLR-mediated TNF-to-IL-10 Concentration Ratio in Newborn and Adult Blood}

PTX inhibited LPS-, R848-, and LPS/ATP-induced IL-10 production in newborn blood, but only diminished R848-induced IL-10 at higher concentrations in adult blood, and actually increased 


\section{Articles | speretal.}

Table 1. PTX inhibited TLR and/or inflammasome agonist-mediated inflammatory cytokine production with higher potency in newborn compared with adult blood

\begin{tabular}{|c|c|c|c|c|}
\hline \multirow[b]{3}{*}{ Cytokine } & \multirow[b]{3}{*}{ TLR and/or inflammasome agonist } & \multirow[b]{3}{*}{$\operatorname{PTX}(\mu \mathrm{mol} / \mathrm{l})$} & \multicolumn{2}{|c|}{ Cytokine conc. (\%) / no PTX $=100 \%$} \\
\hline & & & \multicolumn{2}{|c|}{ Mean $(95 \% \mathrm{Cl})^{\mathrm{a}}$} \\
\hline & & & Newborns $(n=10)$ & Adults $(n=10)$ \\
\hline \multirow[t]{8}{*}{ TNF } & LPS & 50 & $-63.75(-69.99,-57.50)$ & $-38.71(-44.96,-32.47)$ \\
\hline & & 200 & $-89.89(-96.13,-83.64)$ & $-77.07(-83.32,-70.83)$ \\
\hline & & 400 & $-95.76(-102.0,-89.51)$ & $-91.12(-97.37,-84.88)$ \\
\hline & & 200 & $-77.96(-86.72,-69.20)$ & $-44.96(-53.72,-36.20)$ \\
\hline & & 400 & $-93.43(-102.2,-84.68)$ & $-70.16(-78.92,-61.40)$ \\
\hline & LPS/ATP & 50 & $-66.20(-71.19,-61.21)$ & $-34.12(-39.11,-29.13)$ \\
\hline & & 100 & $-81.66(-86.65,-76.67)$ & $-55.90(-60.89,-50.91)$ \\
\hline & & 200 & $-91.04(-96.02,-86.05)$ & $-76.53(-81.52,-71.55)$ \\
\hline \multirow{10}{*}{ IL-1 $\beta$} & & 200 & $-48.27(-56.86,-39.68)$ & $-49.34(-57.93,-40.75)$ \\
\hline & & 400 & $-45.51(-54.10,-36.92)$ & $-63.45(-72.04,-54.86)$ \\
\hline & R848 & 50 & $-27.96(-37.82,-18.09)$ & $-9.68(-19.55,0.18)$ \\
\hline & & 100 & $-43.65(-53.51,-33.78)$ & $-18.04(-27.90,-8.17)$ \\
\hline & & 200 & $-62.38(-72.25,-52.52)$ & $-29.15(-39.02,-19.29)$ \\
\hline & & 400 & $-77.32(-87.18,-67.45)$ & $-53.55(-63.42,-43.68)$ \\
\hline & LPS/ATP & 50 & $-19.21(-33.62,-4.81)$ & $-14.11(-28.52,0.29)$ \\
\hline & & 100 & $-29.55(-43.96,-15.14)$ & $-28.06(-42.46,-13.65)$ \\
\hline & & 200 & $-34.62(-49.02,-20.21)$ & $-26.51(-40.92,-12.10)$ \\
\hline & & 400 & $-23.58(-37.99,-9.17)$ & $-32.16(-46.57,-17.75)$ \\
\hline \multirow[t]{6}{*}{ IL-6 } & LPS & 50 & $-22.01(-30.51,-13.51)$ & $-16.71(-25.21,-8.21)$ \\
\hline & & 400 & $-45.68(-57.78,-33.58)$ & $-10.40(-22.50,1.70)$ \\
\hline & LPS/ATP & 0.50 & $-27.81(-36.20,-19.42)$ & $-21.87(-30.26,-13.48)$ \\
\hline & & 0.100 & $-43.86(-52.25,-35.48)$ & $-32.70(-41.09,-24.31)$ \\
\hline & & 0.200 & $-46.48(-54.87,-38.09)$ & $-43.79(-52.18,-35.40)$ \\
\hline & & 0.400 & $-53.23(-61.62,-44.84)$ & $-53.43(-61.82,-45.04)$ \\
\hline \multirow[t]{12}{*}{ IL-10 } & LPS & 0.50 & $-18.48(-34.51,-2.45)$ & $12.41(-3.62,28.44)$ \\
\hline & & 0.100 & $-35.94(-51.97,-19.91)$ & $22.59(6.55,38.62)$ \\
\hline & & 0.200 & $-47.90(-63.93,-31.87)$ & $25.20(9.17,41.23)$ \\
\hline & & 0.400 & $-61.47(-77.50,-45.44)$ & $-1.26(-17.29,14.77)$ \\
\hline & R848 & 0.50 & $-7.60(-14.90,-0.30)$ & $1.06(-6.25,8.36)$ \\
\hline & & 0.100 & $-22.46(-29.76,-15.16)$ & $-3.07(-10.38,4.23)$ \\
\hline & & 0.200 & $-42.17(-49.48,-34.87)$ & $-13.14(-20.44,-5.84)$ \\
\hline & & 0.400 & $-65.95(-73.26,-58.65)$ & $-34.70(-42.00,-27.40)$ \\
\hline & LPS/ATP & 0.50 & $-17.27(-38.04,3.50)$ & $28.97(8.20,49.74)$ \\
\hline & & 0.100 & $-31.33(-52.10,-10.56)$ & $37.92(17.15,58.69)$ \\
\hline & & 0.200 & $-45.79(-66.56,-25.02)$ & $58.18(37.41,78.95)$ \\
\hline & & 0.400 & $-57.05(-77.82,-36.28)$ & $31.52(10.75,52.29)$ \\
\hline
\end{tabular}

$\mathrm{Cl}$, confidence interval; IL, interleukin; PTX, pentoxifylline; TLR, Toll-like receptor; TNF, tumor necrosis factor.

aSignificant changes of cytokine concentrations in PTX-treated newborn or adult blood compared with the corresponding untreated newborn or adult samples based on $t$-tests were indicated in bold. 
a

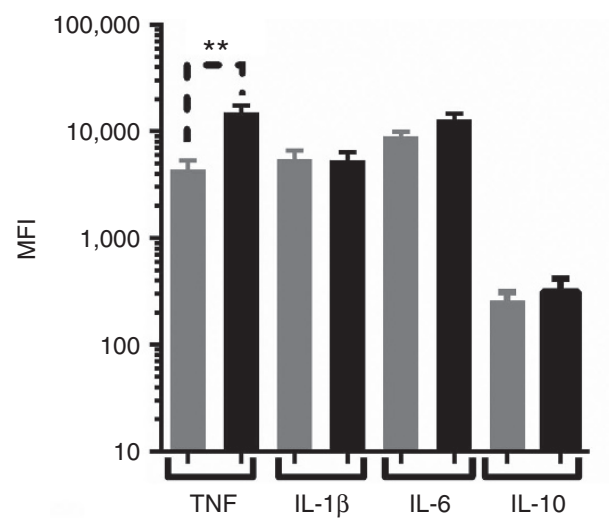

C

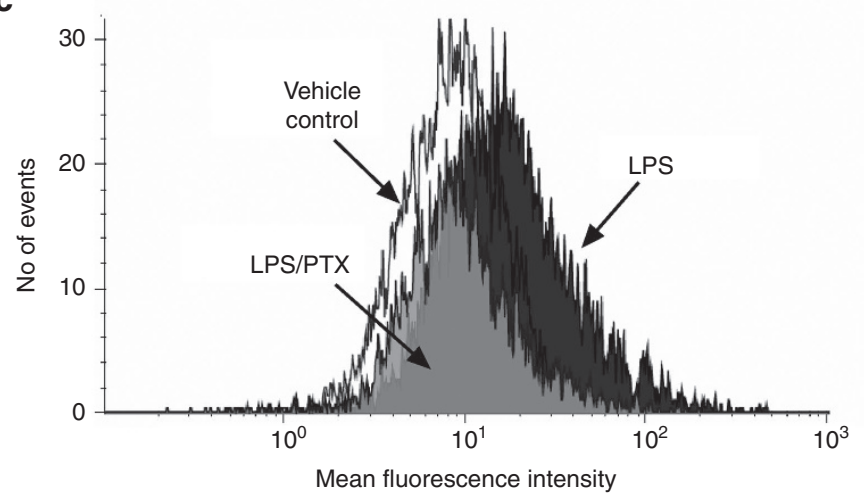

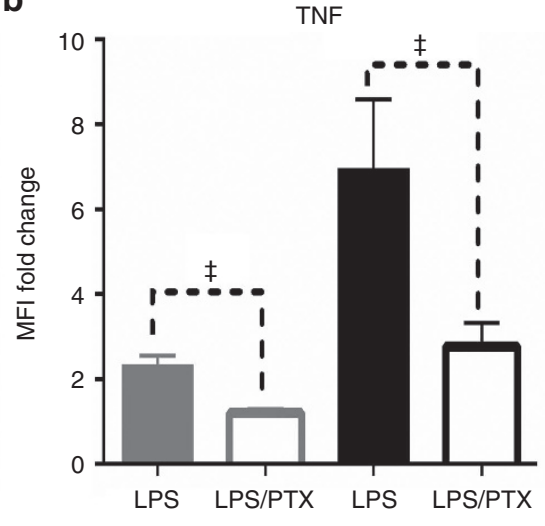

IL-1 $\beta$

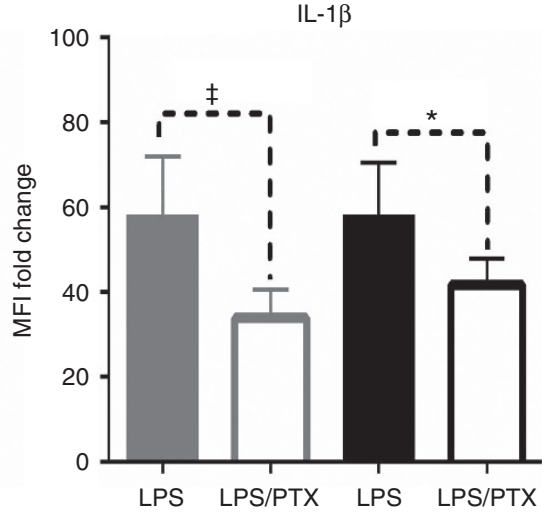

d

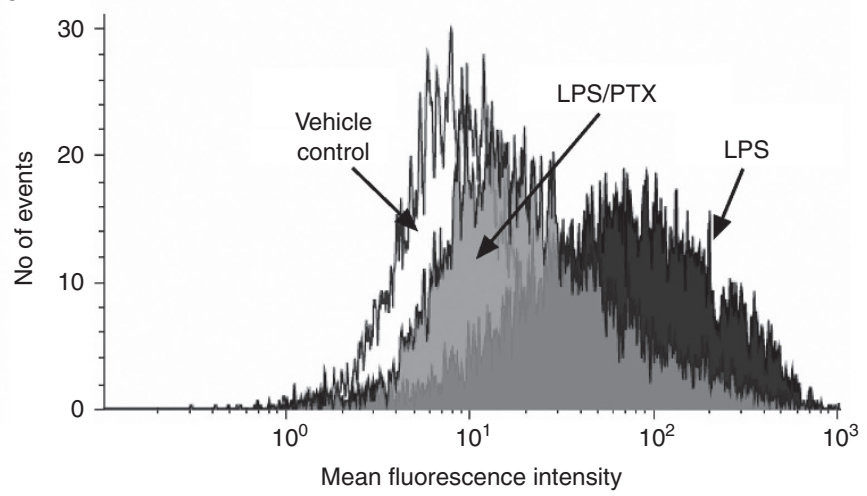

Figure 3. Addition of PTX to whole blood inhibited LPS-induced monocytic intracellular TNF and IL-1 $\beta$. Cord $(n=5)$ and adult blood $(n=5)$, diluted $1: 1$ with RPMI 1640, was pretreated with $200 \mu \mathrm{mol} / \mathrm{I} \mathrm{PTX}$ or vehicle control for $2 \mathrm{~h}$. Samples were stimulated with $10 \mathrm{ng} / \mathrm{ml}$ LPS, and cultured for $6 \mathrm{~h}$ at $37^{\circ} \mathrm{C}$ in $5 \% \mathrm{CO}_{2}$ in the presence (TNF, IL-6, and IL-10) or absence (IL-1 $\beta$ ) of Brefeldin A. After multicolor staining for flow cytometry as described in Methods, MFI data from $\mathrm{CD} 45^{+} / \mathrm{CD} 14^{+}$monocytes, gated on forward and side scatter, were acquired. (a) MFI ( \pm SEM) values (after subtraction of isotype controls) of LPS-induced intracellular cytokines in newborn $(\square)$ vs. adult monocytes $(\square)$. (b) Effects of PTX on LPS-induced intracellular TNF and IL-1 $\beta$ in newborn ( $\square$ ) and adult monocytes $(\square)$, plotted as MFI fold changes compared with unstimulated controls. (c) and (d) Representative histogram of MFI for LPS-induced intracellular TNF in $\mathrm{CD}_{4} 5^{+} / \mathrm{CD}_{1} 4^{+}$(c) newborn and (d) adult blood monocytes in the presence or absence of PTX. Data were analyzed with linear mixed models and significant differences were indicated: ${ }^{*} P<0.05,{ }^{* *} P<0.01,{ }^{*} P<0.001$. ATP, adenosine triphosphate; IL, interleukin; MFI, mean fluorescence intensity; RPMI, Roswell Park Memorial Institute; PTX, pentoxifylline; TNF, tumor necrosis factor.

LPS- and LPS/ATP-induced IL-10 in adult samples (Table 1). Of note, PTX preferentially inhibited TLR-mediated TNF compared with IL-10 production, such that the net effect of PTX was to decrease LPS-, R848-, and LPS/ATP-induced TNF-to-IL-10 concentration ratios in both newborn and adult blood (Figure 2b).

\section{PTX Selectively Inhibited TLR-mediated Intracellular Inflammatory Cytokines in Blood Monocytes}

Newborn monocytes demonstrated significantly lower LPS-induced intracellular TNF expression compared with adult cells (Figure 3a), which was not evident for R848 (see Supplementary Figure S4a online). Pretreatment with PTX decreased LPS- (Figure 3b) and R848-induced (see Supplementary Figure S4b online) intracellular TNF and IL-1 $\beta$ in newborn and adult monocytes, but did not affect intracellular IL-6 and IL-10.

\section{Effect of PTX on TLR-mediated Cytokine Production in Newborn} and Adult Blood was Independent of Timing

Next we determined if delayed PTX addition to established inflammation as compared with pretreatment or simultaneous administration would still be effective. Figure 4 a shows the sequence and duration of sample stimulation and treatment. PTX (50 or 200 $\mu \mathrm{mol} / \mathrm{l})$ diminished LPS- and R848-induced TNF, IL-1 $\beta$, and IL-6 concentrations in blood samples treated $2 \mathrm{~h}$ before, simultaneously or $2 \mathrm{~h}$ after TLR stimulation (Figure $4 \mathrm{~b}, \mathrm{c}$ ), albeit with diminished efficacy and potency with delayed treatment. Delay of PTX for $4 \mathrm{~h}$, however, eliminated its anti-inflammatory effects on R848-induced cytokine production and greatly reduced its inhibition of LPSinduced cytokines (see Supplementary Figure S5 online).

\section{Neonatal Plasma Factors Enhanced the Inhibition of LPS-induced TNF Production by PTX}

To determine whether the distinct composition of human newborn cord blood plasma might contribute to the robust anti-inflammatory efficacy of PTX, newborn and adult hemocytes were resuspended in autologous or heterologous plasma (Figure 5a) as previously described (14). As demonstrated in Figure $5 b$, neonatal plasma enhanced the inhibition of LPSinduced TNF production in newborn but not in adult hemocytes. By contrast, neither neonatal nor adult plasma factors modulated the TNF-suppressive effects of db-cAMP (Figure 5c). 
a

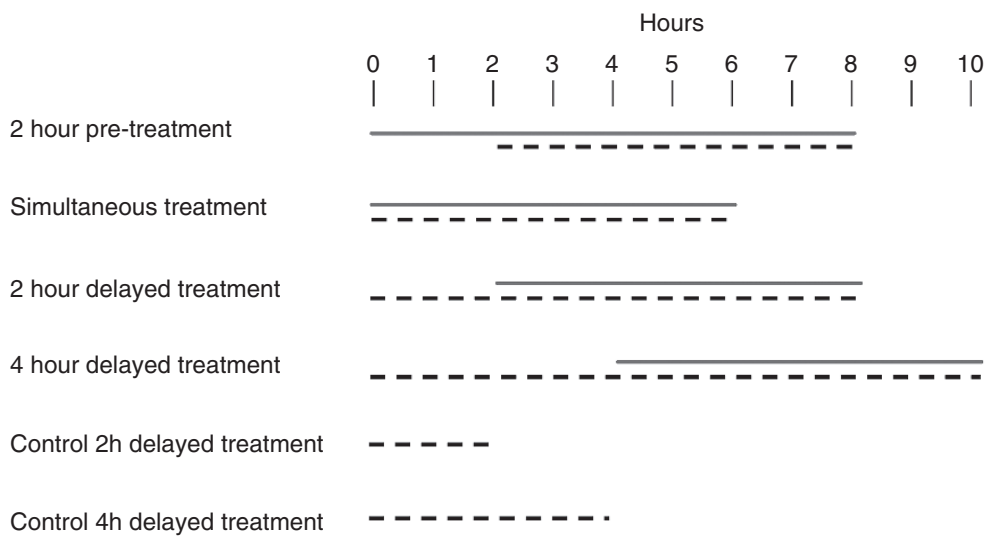

b

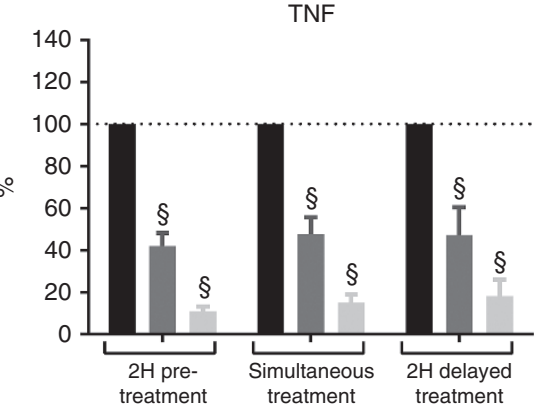

C

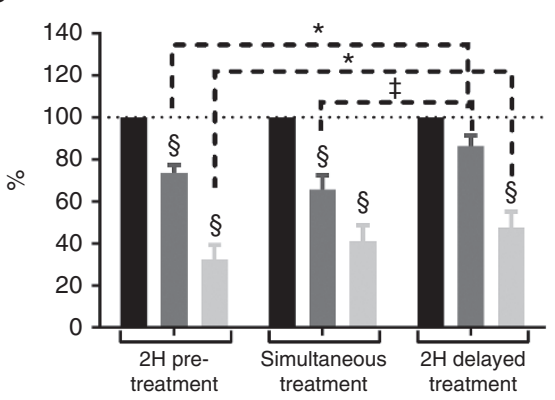

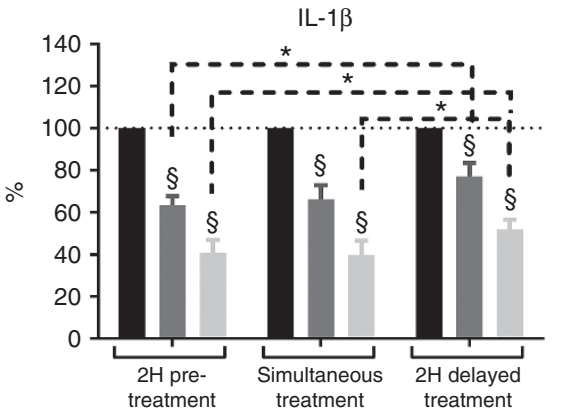

IL-1 $\beta$

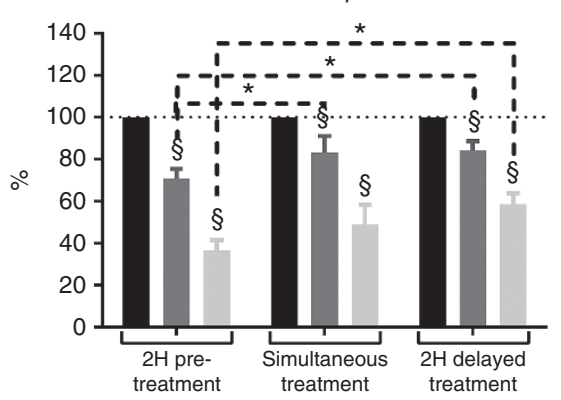

IL-6

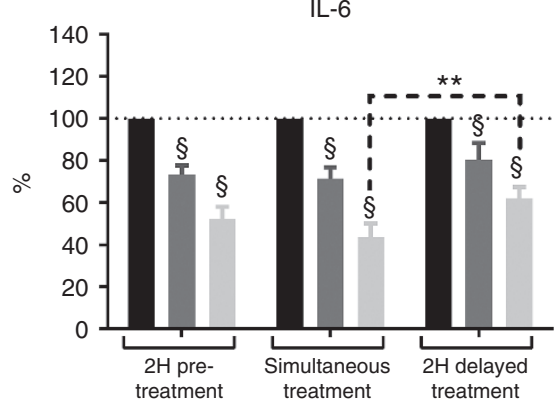

IL-6

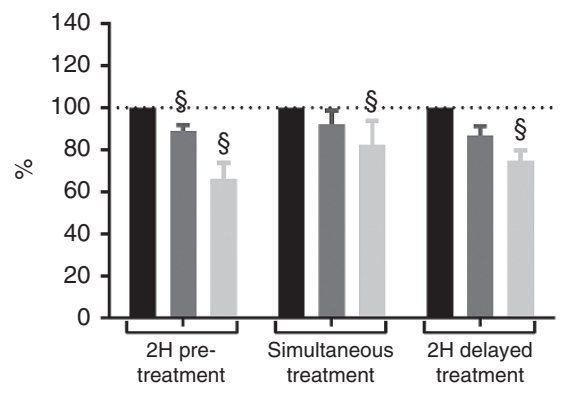

Figure 4. PTX inhibited cytokine production in newborn and adult blood whether added pre-, during, or post-TLR agonist stimulation. Newborn ( $n=5$ ) and adult blood $(n=5)$, diluted $1: 1$ with RPMI 1640, was stimulated with $10 \mathrm{ng} / \mathrm{ml}$ LPS or $1 \mu \mathrm{g} / \mathrm{ml}$ R848. Samples were treated with vehicle control or 50 or $200 \mu \mathrm{mol} / \mathrm{I}$ PTX, added either $2 \mathrm{~h}$ before, simultaneously, or $2 \mathrm{~h}$ or $4 \mathrm{~h}$ after TLR stimulation. Following LPS or R848 addition, samples were cultured for further $6 \mathrm{~h}$. Supernatant cytokines were expressed in percent compared with TLR agonists alone, defined as $100 \%$. For delayed treatment samples, cytokine concentrations measured at the start of anti-inflammatory treatment were subtracted from the corresponding delayed treatment samples, in order not to underestimate the delayed treatment effects. (a) Sequence and duration of stimulation and treatment (anti-inflammatory treatment with PTX: —-_, inflammatory stimulation with LPS or R848: - - ). Effects of timing and concentration (mean \pm SEM) of PTX (no PTX: $\square, 50 \mu$ mol/I PTX: $\square, 200$ mol/I PTX: $\square$ ) on (b) LPS- and (c) R848-induced cytokine production, respectively, in newborn and adult blood combined. A linear mixed model was performed to analyze each cytokine and stimulation independently. The covariance structure between PTX concentrations within a subject was modeled as unstructured variance, and the covariance structure among time points within the same concentration was modeled as compound symmetry. Significant differences between time points were indicated: ${ }^{*} P<0.05,{ }^{* *} P<0.01,{ }^{\ddagger} P<0.001$. Significant decreases in cytokine production of PTX-treated vs. untreated samples, calculated from log transformed raw pg/ml values, were indicated as §. PTX, pentoxifylline; RPMI, Roswell Park Memorial Institute; TLR, Toll-like receptor.

LPS-mediated soluble TNF receptors I (TNF-RI) and II (TNFRII), although present at significantly higher concentrations in newborn compared with adult blood (see Supplementary Figure S6a online), were not altered in response to increasing PTX concentrations (see Supplementary Figure S6b online).

\section{PTX Decreased TLR Agonist-induced TNF mRNA while Enhancing} IL10 mRNA in Whole Blood

TLR agonists induced expression of $m R N A s$ encoding inflammatory cytokines, nuclear factor карра B (NFkB)-, and IkB-related genes, whereas TLR gene expression decreased and IL-10 mRNA remained unchanged (see Supplementary Table S1 online). PTX down-regulated TLR agonist- and inflammasome-induced TNF mRNA and significantly increased IL-10 mRNA at $1 \mathrm{~h}$ and 2h (Figure 6, Supplementary Table S2 online). TLR-agonist induced pro-IL-1 $\beta$ and IL- 6 mRNA increased transiently in response to PTX (Figure 6, Supplementary Table S2 online). Of note, PTX demonstrated little if any inhibition of LPS- or R848induced TLR, NFkB or IkB $\alpha$ expression (see Supplementary Figure S7 online and Supplementary Table S2 online). 
a

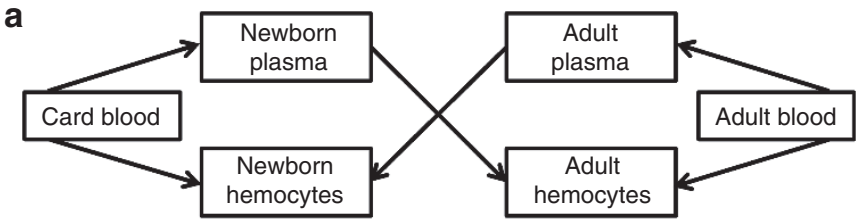

b

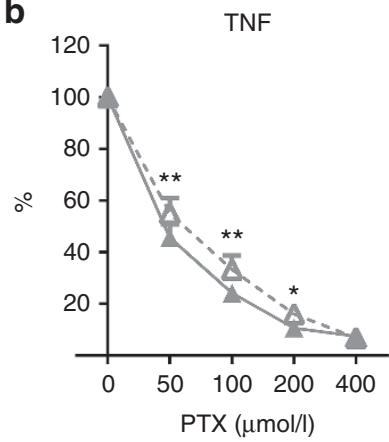

C

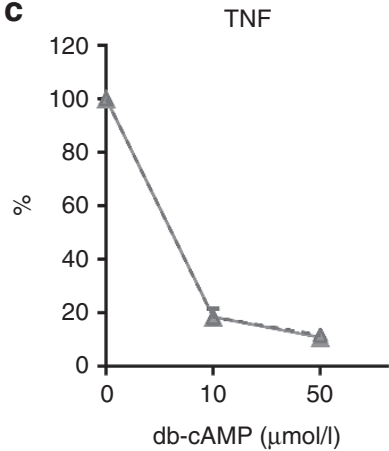

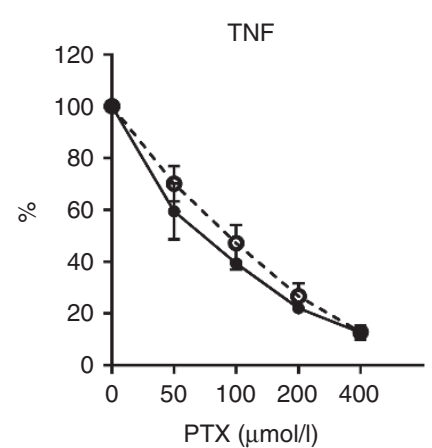

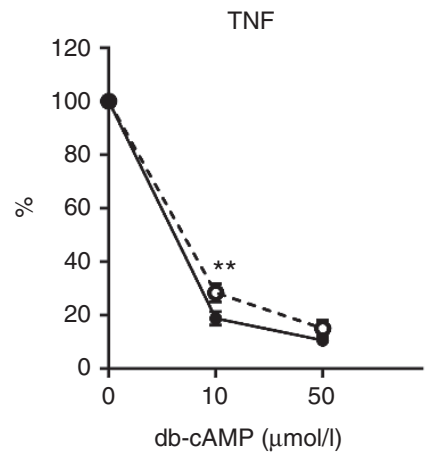

Figure 5. Soluble factors in neonatal plasma augmented the TNFsuppressive actions of PTX. (a) Hemocytes were isolated from cord or adult plasma by centrifugation and washed three times in Hanks' Buffered Salt Solution. Neonatal or adult hemocytes were resuspended in 100\% autologous or heterologous plasma. Samples were pretreated with PTX, db-cAMP, or vehicle control for $2 \mathrm{~h}$, stimulated with $10 \mathrm{ng} / \mathrm{ml}$ LPS and cultured for $6 \mathrm{~h}$, followed by harvesting of supernatants for cytokine measurements. A linear mixed model was performed to analyze newborn and adult cohorts and treatments independently. Anti-inflammatory concentration was modeled as a continuous variable. The covariance structure between homologous and heterologous samples within a subject was modeled as unstructured variance, and the covariance structure among doses within the same homologous and heterologous sample was modeled as compound symmetry. Comparisons of mean LPS-induced TNF concentrations in response to equimolar PTX or db-cAMP among homologous and heterologous samples employed $t$-tests ( $\left(P<0.05,{ }^{* * *} P<0.01,{ }^{*} P<0.001\right)$, while $F$-tests compared their overall drug response curves. (b) and (c) illustrate the concentration-dependent effects of PTX and db-cAMP, respectively, on the inhibition of LPS-induced TNF production (mean \pm SEM) in newborn ( $\Delta$ ) and adult homologous $(\bullet)$ and newborns $(\Delta)$ and adult heterologous $(O)$ blood in percent, with LPS-stimulation alone defined as $100 \%$. Inhibition by PTX of LPS-induced TNF production in homologous vs. heterologous newborn ( $F$-test: $P<0.05)$ and adult blood ( $F$-test: NS), inhibition by db-cAMP of LPS-induced TNF production in homologous vs. heterologous newborn (F-test: NS) and adult blood (F-test: NS). PTX, pentoxifylline; TNF, tumor necrosis factor; TLR, Toll-like receptor.

\section{PTX did not Alter p38 MAPK Phosphorylation and ІкB $\alpha$} Degradation in Newborn and Adult Monocytes

Newborn monocytes demonstrated diminished LPS- and R848-induced total $\operatorname{IkB} \alpha$ (see Supplementary Figure S8a online). Whereas LPS and R848 induced p38 MAPK

phosphorylation and degradation of total $\mathrm{IkB} \alpha$ in newborn and adult monocytes, addition of PTX did not change these parameters (see Supplementary Figure S8b online).

\section{DISCUSSION}

Our study characterized the ability of PTX to modulate TLRmediated cytokine production in human newborn and adult whole blood tested in vitro. Neonatal inflammation is driven in part by TLR signaling and can contribute to host defense against infection (27-30). However, TLR-mediated cytokine induction can also contribute to pathology and disease, including inflammatory diseases of early life such as sepsis, BPD and perinatal brain injury (31-33). PTX, a nonspecific phosphodiesterase inhibitor that decreases transcription of proinflammatory cytokines, is a promising anti-inflammatory candidate for neonates. Based on our prior reports regarding the sensitivity of newborn leukocytes to cAMP axis inhibition (14), we hypothesized that PTX may decrease TLR-mediated cytokine production with greater efficacy in human newborns compared with adults.

In agreement with prior studies, we noted a pattern of diminished LPS- and LPS/ATP-induced but high R848induced TNF and TLR-mediated IL-10 production in cord blood $(25,26)$. Our study utilized whole cord blood to study the effects of PTX on neonatal inflammatory cytokine production. To further maximize the relevance of our in vitro study, PTX was used within clinically relevant concentrations (34). For example, administration of $60 \mathrm{mg} / \mathrm{kg} / \mathrm{d}$ PTX as continuous intravenous infusion over $12 \mathrm{~h}$ for three consecutive days in septic preterm neonates was well-tolerated and resulted in measured serum concentrations of $51.7 \pm 18.1$ $\mu \mathrm{mol} / \mathrm{l}$ PTX (34). Consistent with our findings, clinical trials of adjunctive PTX in neonatal sepsis have employed doses of $30-60 \mathrm{mg} / \mathrm{kg} / \mathrm{d}$ PTX $(8,12)$, associated with significant reduction of serum TNF concentrations (8).

We demonstrated that PTX inhibited LPS- and R848induced TNF and R848-induced IL- $1 \beta$ and IL- 6 production more efficaciously and potently in human newborn compared with adult blood. PTX inhibition of LPS-induced TNF was significantly greater than that of R848, suggesting stimulus-dependent anti-inflammatory responses. PTX inhibition of the NFkB pathway (TNF) was also greater than inhibition of the inflammasome pathway (IL-1 $\beta$ ). Furthermore, PTX decreased the LPS-, R848-, and LPS/ATP-induced TNFto-IL-10 concentration ratios in newborn and adult blood at all tested PTX concentrations. These findings are in line with the previously reported differential regulation of LPS- or PHA-induced proinflammatory cytokines and IL-10 by PTX and db-cAMP $(35,36)$. Our study extended the findings on cytokine regulation by PTX to TLR7/8 and inflammasome pathways. Although PTX inhibited LPS-induced IL-10 in newborn and R848-induced IL-10 production in newborn and adult samples, it did so to a lesser extent than toward TNF production, with a net effect of reducing the TNFto-IL-10 ratio. Indeed, PTX increased TLR-mediated IL-10 mRNA expression would be consistent with this explanation. 


\section{Articles | speretal.}

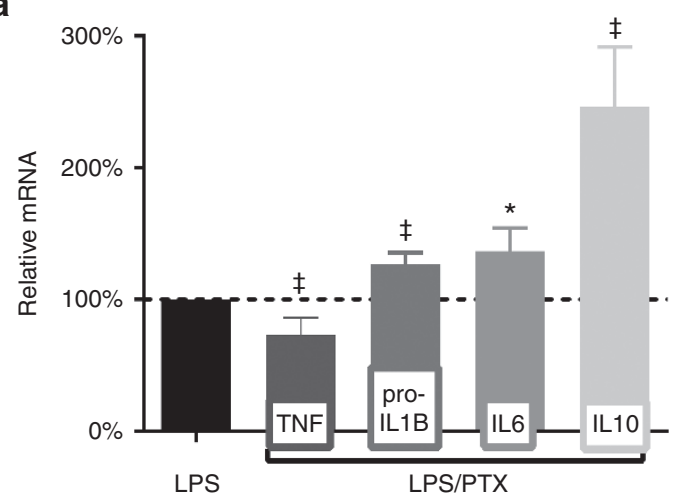

b

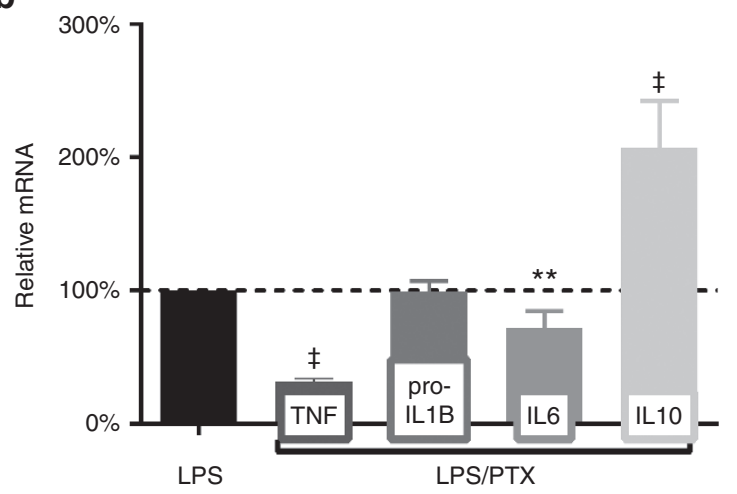

Figure 6. PTX selectively decreased LPS-induced proinflammatory TNF mRNA in whole blood while increasing anti-inflammatory IL-10 gene expression. Cord $(n=5)$ and adult blood $(n=5)$, diluted $1: 1$ with RPMI 1640, was pretreated with $200 \mu$ mol/I PTX or vehicle control for $2 \mathrm{~h}$ and cultured for $1 \mathrm{~h}$ (a) or $2 \mathrm{~h}(\mathbf{b})$ in the presence of $10 \mathrm{ng} / \mathrm{ml}$ LPS. Total RNA was isolated from whole blood, reverse transcribed, and relative $\mathrm{mRNA}$ expression (mean \pm SEM) in response to PTX compared with LPS alone, defined as $100 \%$, was determined from $\Delta \Delta C_{T}$ values with real time PCR. Results for newborn and adult samples were combined $(n=10)$. Significant differences between LPS-stimulated samples that were pretreated or not with $\mathrm{PTX}$ were indicated: ${ }^{*} P<0.05$, ${ }^{* *} P<0.01$, ${ }^{\ddagger} P<0.001$. IL, interleukin; RPMI, Roswell Park Memorial Institute; PTX, pentoxifylline; TNF, tumor necrosis factor; TLR, Toll-like receptor.

Although we did not observe enhancement of IL-10 production by PTX $(50-400 \mu \mathrm{mol} / \mathrm{l})$ in unstimulated blood, very high concentrations of PTX (1 $\mathrm{mmol} / \mathrm{l})$ were reported to increase IL-10 in adult blood (36). PTX may thus in a stimulus- and concentration-dependent manner enhance IL-10 production.

Our findings on intracellular cytokines in monocytes were consistent with whole blood supernatant cytokine results. PTX decreased TLR-mediated intracellular TNF and IL- $1 \beta$ in newborn and adult monocytes while IL- 6 and IL- 10 remained unchanged, confirming the differential effects of this agent on pro- and anti-inflammatory cytokine production (35).

PTX effectively reduced inflammatory cytokines in newborn and adult blood when used before, simultaneously, or $2 \mathrm{~h}$ after TLR stimulation. To the extent that our in vitro data mirror conditions that may be relevant in vivo, this agent may thus be used both prophylactically as well as therapeutically for the treatment of inflammation. Experiments in vivo, both in animal sepsis models and in human clinical trials, however, will be required to assess the potential beneficial impact of PTX on inflammation and disease.

PTX decreased TLR agonist- and inflammasome-induced TNF mRNA while enhancing IL-10 mRNA. This suggests that PTX exerts its effects through transcriptional modification of pro and anti-inflammatory cytokines, which is consistent with its cAMP-dependent anti-inflammatory pathway $(5,6,35)$.

Newborn monocytes demonstrated diminished LPS- and R848-induced total $\mathrm{I} k \mathrm{~B} \alpha$ phosphorylation compared with adult cells, as previously described (37). Whereas LPS and R848 both increased p38 MAPK phosphorylation and $\mathrm{IkB} \alpha$ degradation in newborn and adult monocytes, PTX did not change these parameters. This suggests that PTX exerts its immunomodulatory effects either downstream and/or independently of these inflammatory pathways.

Consistent with the previously reported anti-inflammatory effects of neonatal plasma (16), reflecting in part the contribution of adenosine (14), our experiments suggest that neonatal plasma enhanced the inhibition of LPS-induced TNF production in newborn but not in adult hemocytes. By contrast, neither neonatal nor adult plasma modulated the TNF-suppressive effects of db-cAMP, suggesting that neonatal plasma might enhance the anti-inflammatory actions of PTX either upstream or independently of intracellular cAMP.

Soluble cytokine receptors, which act as endogenous antiinflammatory factors, are elevated in cord and newborn blood during the perinatal period $(16,38)$. Our findings of elevated soluble receptors TNF-RI and TNF-RII in newborn blood plasma are in line with these reports. However, soluble TNF receptors were not responsible for the greater anti-inflammatory efficacy of PTX in newborn blood.

Our in vitro experiments were conducted on whole blood from adults and healthy term neonates delivered without evidence of inflammation and in the absence of labor. Future studies should explore the anti-inflammatory responses to PTX in blood obtained from additional clinically relevant target populations including newborns delivered vaginally (as labor may influence the inflammatory response and its modulation by PTX), neonates exposed to intrauterine infection and inflammation, septic newborns, and neonates delivered preterm. Additionally, samples from other anatomic compartments that are commonly affected by exaggerated inflammatory responses, such as tracheal aspirate samples from ventilated neonates (39), might be considered.

In summary, our study utilized TLR and/or inflammasome agonist-stimulated whole cord blood to characterize the anti-inflammatory effects of PTX. We demonstrated that PTX inhibited TLR-mediated inflammatory cytokine production with higher efficacy and potency in human newborn compared with adult blood while relatively preserving IL-10 responses. Activity toward inflammatory responses triggered via the TLR7/8 pathway activated by R848 suggests potential utility against inflammation induced by bacterial infections harboring bacterial RNA that may induce robust inflammation 
via TLR7/8 (20) as well as that induced by single stranded viruses such as HIV and influenza (19). PTX exerted antiinflammatory effects when used prophylactically or simultaneously to inflammatory stimulation, or when administered in ongoing inflammation. PTX, which supports the neonatal Th2 cytokine pattern characterized by low TNF but high IL-10 and IL-6 $(26,27)$, might thus provide a promising anti-inflammatory therapy for neonates, and indeed larger clinical trials of PTX have recently been initiated in this population (8). To the extent that our in vitro studies mirror effects of PTX in vivo, it may be particularly effective in reducing infection-induced inflammation in the newborn. Indeed, our experience with in vitro assessment of other anti-inflammatory agents such as bactericidal permeability-increasing protein that is active in neutralizing endotoxin in vivo supports the predictive capacity of this assay system (40). Finally, the distinct anti-inflammatory effects of pharmaceutical agents with age, as shown in our experimental model on pattern recognition receptor agoniststimulated newborn and adult blood treated with PTX, suggest that translational development in this sphere should take immune ontogeny into account.

\section{SUPPLEMENTARY MATERIAL}

Supplementary material is linked to the online version of the paper at http:// www.nature.com/pr

\section{ACKNOWLEDGMENTS}

The authors greatly thank Avinash Chander, Department of Pediatrics, and Berhane Ghebrehiwet, Department of Medicine, Stony Brook University School of Medicine, for providing mentorship and critical review of our manuscript. We also thank Jaime Mathew, Department of Pediatrics, Stony Brook University, for technical assistance with whole blood cultures, immunoassays and real time PCR experiments, and members of the Levy Laboratory at Boston Children's Hospital for their technical assistance with LAL experiments. We further acknowledge Todd Rueb, Flow Cytometry Core Facility, Stony Brook University School of Medicine, for assistance with flow cytometry experiments and interpretation of results. Finally, we acknowledge the biostatistical consultation and support provided by the Biostatistical Consulting Core at the School of Medicine, Stony Brook University.

\section{STATEMENT OF FINANCIAL SUPPORT}

E.S.'s laboratory is financially supported by the Little Angel Fund NICU Support Group, Selden, NY, and the Department of Pediatrics Intramural Grant Program, Stony Brook Children's Hospital and Stony Brook University School of Medicine, Stony Brook, NY. O.L.'s laboratory is supported by US National Institutes of Health (NIH) grant R01Al100135-01 and its administrative supplements as well as National Institutes of Allergy and Infectious Diseases (NIAID) Adjuvant Discovery Program Contract No. HHSN272201400052C. The funding bodies did not play any role in the study design, in the collection, analysis and interpretation of data, in the writing of the manuscript, and in the decision to submit the manuscript for publication.

Disclosure: The authors confirm that there are no financial ties to products in the study or conflicts of interest to disclose.

\section{REFERENCES}

1. Kuban KC, O'Shea TM, Allred EN, et al.; ELGAN Study Investigators. Systemic inflammation and cerebral palsy risk in extremely preterm infants. J Child Neurol 2014;29:1692-8.

2. Cornette L. Fetal and neonatal inflammatory response and adverse outcome. Semin Fetal Neonatal Med 2004;9:459-70.

3. Halliday HL, Ehrenkranz RA, Doyle LW. Early ( $<8$ days) postnatal corticosteroids for preventing chronic lung disease in preterm infants. Cochrane Database Syst Rev 2014, CD001146.
4. Halliday HL, Ehrenkranz RA, Doyle LW. Late (>7 days) postnatal corticosteroids for chronic lung disease in preterm infants. Cochrane Database Syst Rev 2014, CD001145.

5. Serezani CH, Ballinger MN, Aronoff DM, Peters-Golden M. Cyclic AMP: master regulator of innate immune cell function. Am J Respir Cell Mol Biol 2008;39:127-32.

6. Minguet S, Huber M, Rosenkranz L, Schamel WW, Reth M, Brummer T. Adenosine and cAMP are potent inhibitors of the NF-kappa B pathway downstream of immunoreceptors. Eur J Immunol 2005;35:31-41.

7. Harris E, Schulzke SM, Patole SK. Pentoxifylline in preterm neonates: a systematic review. Paediatr Drugs 2010;12:301-11.

8. Shabaan AE, Nasef N, Shouman B, Nour I, Mesbah A, Abdel-Hady H. Pentoxifylline therapy for late-onset sepsis in preterm infants: a randomized controlled trial. Pediatr Infect Dis J 2015;34:e143-8.

9. Schulzke SM, Kaempfen S, Patole SK. Pentoxifylline for the prevention of bronchopulmonary dysplasia in preterm infants. Cochrane Database Syst Rev 2014, CD010018.

10. Turhan AH, Atıcı A, Muşlu N, Polat A, Helvacı İ. The effects of pentoxifylline on lung inflammation in a rat model of meconium aspiration syndrome. Exp Lung Res 2012;38:250-5.

11. Kalay S, Oztekin O, Tezel G, et al. The effects of intraperitoneal pentoxifylline treatment in rat pups with hypoxic-ischemic encephalopathy. Pediatr Neurol 2013;49:319-23.

12. Pammi M, Haque KN. Pentoxifylline for treatment of sepsis and necrotizing enterocolitis in neonates. Cochrane Database Syst Rev 2015, CD004205.

13. Wynn JL, Wong HR, Shanley TP, Bizzarro MJ, Saiman L, Polin RA. Time for a neonatal-specific consensus definition for sepsis. Pediatr Crit Care Med 2014;15:523-8.

14. Levy O, Coughlin M, Cronstein BN, Roy RM, Desai A, Wessels MR. The adenosine system selectively inhibits TLR-mediated TNF-alpha production in the human newborn. J Immunol 2006;177:1956-66.

15. Philbin VJ, Dowling DJ, Gallington LC, et al. Imidazoquinoline Toll-like receptor 8 agonists activate human newborn monocytes and dendritic cells through adenosine-refractory and caspase-1-dependent pathways. J Allergy Clin Immunol 2012;130:195-204.e9.

16. Belderbos ME, Levy O, Meyaard L, Bont L. Plasma-mediated immune suppression: a neonatal perspective. Pediatr Allergy Immunol 2013;24:10213.

17. Elahi S, Ertelt JM, Kinder JM, et al. Immunosuppressive CD71+ erythroid cells compromise neonatal host defence against infection. Nature 2013;504:158-62.

18. Dowling DJ, Levy O. Ontogeny of early life immunity. Trends Immunol 2014;35:299-310.

19. Philbin VJ, Levy O. Immunostimulatory activity of Toll-like receptor 8 agonists toward human leucocytes: basic mechanisms and translational opportunities. Biochem Soc Trans 2007;35(Pt 6):1485-91.

20. Eigenbrod T, Pelka K, Latz E, Kreikemeyer B, Dalpke AH. TLR8 senses bacterial RNA in human monocytes and plays a nonredundant role for recognition of streptococcus pyogenes. J Immunol 2015;195:1092-9.

21. Gibbs RS, Blanco JD, St Clair PJ, Castaneda YS. Quantitative bacteriology of amniotic fluid from women with clinical intra-amniotic infection at term. J Infect Dis 1982;145:1-8.

22. Dowling DJ, Tan Z, Prokopowicz ZM, et al. The ultra-potent and selective TLR8 agonist VTX-294 activates human newborn and adult leukocytes. PLoS One 2013;8:e58164.

23. Pestana EA, Belak S, Diallo A, Crowther JR, Viljoen GJ. Early, Rapid and Sensitive Veterinary Molecular Diagnostics-Real Time PCR Applications. Dordrecht, Netherlands: Springer Science \& Business Media, 2010: 247-63.

24. Bender R, Lange S. Adjusting for multiple testing-when and how? J Clin Epidemiol 2001;54:343-9.

25. Levy O, Zarember KA, Roy RM, Cywes C, Godowski PJ, Wessels MR. Selective impairment of TLR-mediated innate immunity in human newborns: neonatal blood plasma reduces monocyte TNF-alpha induction by bacterial lipopeptides, lipopolysaccharide, and imiquimod, but preserves the response to R-848. J Immunol 2004;173:4627-34. 


\section{Articles | speeretal.}

26. Angelone DF, Wessels MR, Coughlin M, et al. Innate immunity of the human newborn is polarized toward a high ratio of IL-6/TNF-alpha production in vitro and in vivo. Pediatr Res 2006;60:205-9.

27. Kollmann TR, Levy O, Montgomery RR, Goriely S. Innate immune function by Toll-like receptors: distinct responses in newborns and the elderly. Immunity 2012;37:771-83.

28. Pettengill MA, van Haren SD, Levy O. Soluble mediators regulating immunity in early life. Front Immunol 2014;5:457.

29. Corbett NP, Blimkie D, Ho KC, et al. Ontogeny of Toll-like receptor mediated cytokine responses of human blood mononuclear cells. PLoS One 2010;5:e15041.

30. Burl S, Townend J, Njie-Jobe J, et al. Age-dependent maturation of Tolllike receptor-mediated cytokine responses in Gambian infants. PLoS One 2011;6:e18185.

31. Redondo AC, Ceccon ME, Silveira-Lessa AL, et al. TLR-2 and TLR-4 expression in monocytes of newborns with late-onset sepsis. J Pediatr (Rio J) 2014;90:472-8.

32. Bi D, Qiao L, Bergelson I, et al. Staphylococcus epidermidis bacteremia induces brain injury in neonatal mice via toll-like receptor 2-dependent and -independent pathways. J Infect Dis 2015;212:1480-90.

33. Mallard C, Wang X, Hagberg $H$. The role of Toll-like receptors in perinatal brain injury. Clin Perinatol 2009;36:763-72, v-vi.
34. Szymura-Oleksiak J, Bury J, Lauterbach R, Pawlowski M. Serum concentrations of pentoxifylline and its metabolites in premature infants with sepsis when administered by continuous intravenous infusion. Pharmaceutical Sciences 1997;3:367-71.

35. Benbernou N, Esnault S, Shin HC, Fekkar H, Guenounou M. Differential regulation of IFN-gamma, IL-10 and inducible nitric oxide synthase in human T cells by cyclic AMP-dependent signal transduction pathway. Immunology 1997;91:361-8.

36. D'Hellencourt CL, Diaw L, Cornillet P, Guenounou M. Differential regulation of TNF alpha, IL-1 beta, IL-6, IL-8, TNF beta, and IL-10 by pentoxifylline. Int J Immunopharmacol 1996;18:739-48.

37. Levy O, Suter EE, Miller RL, Wessels MR. Unique efficacy of Toll-like receptor 8 agonists in activating human neonatal antigen-presenting cells. Blood 2006;108:1284-90.

38. Protonotariou E, Rizos D, Malamitsi-Puchner A, Moira E, Sarandakou A, Salamalekis E. Cytokine soluble receptors in perinatal and early neonatal life. Mediators Inflamm 2003;12:185-8.

39. Nathe KE, Mancuso CJ, Parad R, et al. Innate immune activation in neonatal tracheal aspirates suggests endotoxin-driven inflammation. Pediatr Res 2012;72:203-11.

40. Levy O. A neutrophil-derived anti-infective molecule: bactericidal/permeability-increasing protein. Antimicrob Agents Chemother 2000;44:2925-31. 